PROCEEDINGS OF THE

AMERICAN MATHEMATICAL SOCIETY

Volume 139, Number 1, January 2011, Pages 231-242

S 0002-9939(2010)10576-2

Article electronically published on July 23, 2010

\title{
ON LIOUVILLE INTEGRABILITY OF $h$-PROJECTIVELY EQUIVALENT KÄHLER METRICS
}

\author{
KAZUYOSHI KIYOHARA AND PETER TOPALOV
}

(Communicated by Jon G. Wolfson)

\begin{abstract}
Under a nondegeneracy condition we classify the compact connected Kähler manifolds admitting pairs of $h$-projectively equivalent metrics. Any such manifold is biholomorphically equivalent to $\mathbb{C} P^{n}$ and has integrable geodesic flow.
\end{abstract}

\section{INTRODUCTION}

In [5, 6, 8, and [9, pairs of Riemannian metrics whose Levi-Civita connections are mutually projectively equivalent were studied. It was proven, among other things, that under a nondegeneracy condition such Riemannian manifolds have integrable geodesic flows. More specifically, it was shown that these manifolds are Liouville manifolds in the sense of [1]. Also, from a single pair of projectively equivalent metrics, a hierarchy of such pairs was constructed; see e.g. [8] and the references therein. These results were generalized to metrics of arbitrary signature in 9 .

The holomorphic counterpart to this problem was studied in [10. There were considered pairs of holomorphically projectively equivalent Kähler metrics (or simply, $h$-projectively equivalent metrics). By definition, two Kähler metrics $g$ and $g^{\prime}$ are $h$-projectively equivalent if their Levi-Civita connections $\nabla$ and $\nabla^{\prime}$ satisfy

$$
\nabla_{X}^{\prime} Y-\nabla_{X} Y=\phi(X) Y+\phi(Y) X-\phi(J X) J Y-\phi(J Y) J X
$$

where $\phi$ is a one-form and $J$ stands for the complex structure on the manifold (see [4). Equation (1.1) implies that any holomorphically planar curve with respect to one of the metrics is holomorphically planar also with respect to the other metric and vice versa; see for example [10, Introduction, Example (b)], 4, 7], for more details, as well as [11, 12, for related work. It was shown in [10] that under a nondegeneracy condition Kähler manifolds admitting pairs of $h$-projective equivalent metrics are Kähler-Liouville manifolds in the sense of [1]. As in the real case, there exists a hierarchy of such pairs of metrics. However, the metrics appearing in the hierarchy are no longer Kähler, but only Hermitian in general. They satisfy a modified version of (1.1):

$$
\nabla_{X}^{\prime} Y-\nabla_{X} Y=\phi(X) Y+\phi(Y) X-\phi(P X) Q Y-\phi(P Y) Q X,
$$

Received by the editors August 4, 2009 and, in revised form, February 28, 2010.

2010 Mathematics Subject Classification. Primary 37J35, 53D25, 53C55.

The second author was supported in part by NSF grant DMS-0901443.

(C)2010 American Mathematical Society 
where $\phi$ is a one-form on $M ; P$ is a nondegenerate, skew-symmetric with respect to both $g$ and $g^{\prime},(1,1)$-type tensor field; and $Q=-P^{-1}$.

Results. The aim of this paper is twofold. First, we show that under a nondegeneracy condition Kähler manifolds admitting $h$-projectively equivalent metrics are Kähler-Liouville manifolds of a special type (see Theorem 2.2, Section 2). As a consequence, these manifolds have integrable geodesic flows, and, if compact and connected, are biholomorphically equivalent to the complex projective space $\mathbb{C} P^{n}$ (Corollary 2.3. Section 2). From this, one obtains a complete set of invariants that characterize the pairs of $h$-projectively equivalent Kähler metrics (see Theorem 3.3 , Section (3). Second, under a non-degeneracy condition we investigate the local structure of Hermitian manifolds admitting pairs of Hermitian metrics that satisfy the more general equation (1.2). It is shown that such a Hermitian manifold $M$ $\left(\operatorname{dim}_{\mathbb{C}} M=n\right)$ possesses locally an $n$-dimensional real vector space of infinitesimal automorphisms (Theorem 4.6, Section 4).

\section{$2 . h$-PROJECTIVELY EQUIVALENT METRICS AND KäHLER-LIOUVILLE MANIFOLDS}

Let $M$ be a connected complex manifold of complex dimension $n$, and let $g$ and $g^{\prime}$ be two Kähler metrics on $M$ which are mutually $h$-projectively equivalent. As stated in the Introduction, their Levi-Civita connections $\nabla$ and $\nabla^{\prime}$ satisfy

$$
\nabla_{X}^{\prime} Y-\nabla_{X} Y=\phi(X) Y+\phi(Y) X-\phi(J X) J Y-\phi(J Y) J X,
$$

where $J$ denotes the complex structure and $\phi$ is a one-form on $M$. Let $A$ be the (1,1)-type tensor field defined by

$$
g^{\prime}(X, Y)=(\operatorname{det} A)^{-1 / 2} g\left(A^{-1} X, Y\right) .
$$

Then $A$ is symmetric with respect to both $g$ and $g^{\prime}$, and $A J=J A$. Denote by $\mathcal{A}$ the $\mathbb{C}$-linear map corresponding to $A$ when tangent spaces are regarded as complex vector spaces by putting $J=\sqrt{-1}$. Let

$$
h_{1}(p) \geq \cdots \geq h_{n}(p)>0
$$

be the eigenvalues of $\mathcal{A}$ at $p \in M$. Each $h_{i}$ is a continuous function on $M$, and it is smooth at the points $p \in M$ where $h_{i-1}(p)>h_{i}(p)>h_{i+1}(p)$. Note that the eigenvalues of the endomorphism $A$ are $h_{1}, h_{1}, \ldots, h_{n}, h_{n}$. Denote

$$
\chi(c):=\operatorname{det}(\mathcal{A}-c \mathbf{1})=\prod_{i=1}^{n}\left(h_{i}-c\right) .
$$

Proposition 2.1. For any $c \in \mathbb{R}$ consider the function on the tangent bundle $T M$,

$$
K_{c}(X)=\operatorname{det}(\mathcal{A}-c \mathbf{1}) g\left((A-c \mathbf{1})^{-1} X, X\right) \text {. }
$$

Let $K_{c}^{*}$ be the pullback of $K_{c}$ by the Legendre transform $\sharp_{g}: T^{*} M \rightarrow T M$ corresponding to $g$. Then the one-parameter family of functions on $T^{*} M$,

$$
K_{c}^{*}=\sum_{i=1}^{n}(-c)^{i-1} K_{i}
$$

$c \in \mathbb{R}$, consists of Poisson commuting integrals of the geodesic flow of $(M, g) 1$

\footnotetext{
${ }^{1}$ Note that the integrals $K_{1}, \ldots, K_{n}$ appearing in this family are not sufficient for the integrability of the geodesic flow of $g$ as $\operatorname{dim}_{\mathbb{R}} M=2 n$.
} 
For the proof, see [10, Theorem 4, V]. Now, let us make the following assumption.

Assumption 1. There is a point $p_{0} \in M$ such that $\mathcal{A}$ has $n$ distinct eigenvalues at $p_{0}$, i.e., $h_{1}\left(p_{0}\right)>\cdots>h_{n}\left(p_{0}\right)$, and $d h_{i} \neq 0(1 \leq i \leq n)$ at $p_{0}$.

Let $\mathcal{F}$ be the $n$-dimensional vector space of functions on $T^{*} M$ spanned by $K_{1}, \ldots, K_{n}$. Note that $\frac{1}{2} K_{n}$ is the Hamiltonian of the geodesic flow of $(M, g)$.

Theorem 2.2. $(M, g, \mathcal{F})$ is a Kähler-Liouville manifold of type $(A)$ whose associated partially ordered set consists of one element. In particular, there exists an $n$-dimensional vector space $\mathfrak{h}$ of infinitesimal automorphisms of the Kähler manifold $(M, g)$ such that

$$
\left[Y_{1}, Y_{2}\right]=0, \quad\{F, Y\}=0 \quad \text { for any } Y_{1}, Y_{2}, Y \in \mathfrak{h}, F \in \mathcal{F} .
$$

Thus the geodesic flow of $(M, g)$ is integrable with first integrals in $\mathcal{F}$ and $\mathfrak{h}$.

Proof. By definition, $(M, g, \mathcal{F})$ is a Kähler-Liouville manifold if the following conditions are satisfied (see [1, Part 2, Introduction]).

(1) The Hamiltonian $E$ of the geodesic flow belongs to $\mathcal{F}$.

(2) $\{F, H\}=0$ for any $F, H \in \mathcal{F}$.

(3) $F_{p}=\left.F\right|_{T_{p}^{*} M}$ is a Hermitian form for any $p \in M$ and $F \in \mathcal{F}$.

(4) $F_{p}(F \in \mathcal{F})$ are simultaneously normalizable for every $p \in M$.

(5) $\mathcal{F}_{p}=\left\{F_{p} \mid F \in \mathcal{F}\right\}$ is $n$-dimensional at some $p \in M$.

Clearly, $(M, g, \mathcal{F})$ is a Kähler-Liouville manifold. Let $M^{0}$ be the set of all points $p \in M$ where $\mathcal{A}$ has $n$ distinct eigenvalues $h_{1}>\cdots>h_{n}$, and let $M^{1} \subseteq M^{0}$ consists of all points in $M^{0}$, where $d h_{i} \neq 0$ for any $i$.

Take $p_{0} \in M^{1}$ and an orthonormal frame $V_{1}, \ldots, V_{n}, J V_{1}, \ldots, J V_{n}$ of $T M$ on a neighborhood of $p_{0}$ in $M^{1}$ such that $A V_{i}=h_{i} V_{i}, A J V_{i}=h_{i} J V_{i}$. The existence of such a frame follows from $A J=J A$. Then

$$
K_{c}^{*}=\sum_{i=1}^{n}\left(\prod_{j \neq i}\left(h_{j}-c\right)\right)\left(V_{i}^{2}+\left(J V_{i}\right)^{2}\right),
$$

where $V_{i}$ and $J V_{i}$ are regarded as functions on the cotangent bundle 2 As (2.4) holds also when $c$ is a function on $M$ we see that

$$
\sum_{j=1}^{n}\left(-h_{i}\right)^{j-1} K_{j}=\left(\prod_{j \neq i}\left(h_{j}-h_{i}\right)\right)\left(V_{i}^{2}+\left(J V_{i}\right)^{2}\right) .
$$

Using (2.6) and the Poisson commutativity of $K_{1}, \ldots, K_{n}$, we obtain by a direct computation that

$$
\begin{gathered}
\left\{\left(\prod_{k \neq i}\left(h_{k}-h_{i}\right)\right)\left(V_{i}^{2}+\left(J V_{i}\right)^{2}\right),\left(\prod_{l \neq j}\left(h_{l}-h_{j}\right)\right)\left(V_{j}^{2}+\left(J V_{j}\right)^{2}\right)\right\}=0, \\
d h_{j}\left(V_{i}\right)=d h_{j}\left(J V_{i}\right)=0 \quad(j \neq i) .
\end{gathered}
$$

\footnotetext{
${ }^{2}$ Note that under such an identification, $\{X, Y\}=[X, Y]$ and $\{X, f\}=d f(X)$ for any two vector fields $X, Y$ and $f \in C^{\infty}(M)$.
} 
Therefore, the function $a_{i}$ in [1, Proposition 1.1, p. 84] can be taken as

$$
a_{i}=\left((-1)^{n-i} \prod_{k \neq i}\left(h_{k}-h_{i}\right)\right)^{-1} \quad(1 \leq i \leq n) .
$$

Since $d h_{i} \neq 0(1 \leq i \leq n)$, it follows from (2.7) and (2.8) that for any $1 \leq i, j \leq n$, $\left.d a_{i}\right|_{\operatorname{span}\left\{V_{j}, J V_{j}\right\}} \neq 0$. Hence, $(M, g, \mathcal{F})$ is of type (A) and the associated partially ordered set (see [1, Proposition 1.9]) consists of one element. See [1, pp. 84-88] for the definition of Kähler-Liouville manifolds of type (A) and the partial order.

The existence of the space $\mathfrak{h}$ of infinitesimal automorphisms now follows from the general theory of Kähler-Liouville manifolds of type (A); see 1, Proposition 1.16, Theorem 3.1] 3

Now suppose that $M$ is compact and connected. Let $G$ be the transformation group of $M$ generated by $\mathfrak{g}=\mathfrak{h}+J \mathfrak{h}$. The group $G$ is isomorphic to $\left(\mathbb{C}^{\times}\right)^{n}$, and with this action $M$ becomes a toric variety (see 11, Section 4). Since the associated partially ordered set consists of one element, we have the following (cf. [1, Section 7).

Corollary 2.3. As a toric variety, $M$ is isomorphic to the complex projective space $\mathbb{C} P^{n}$.

Remark. As stated in [3], some parts of [1] are incorrect unless an additional condition called properness is assumed. Note however that in the case where the associated partially ordered set consists of one element, the properness condition is automatically satisfied (see $[3$ for more details).

\section{Fundamental inVARIANTS AND ClASSIFICATION}

Let $\left(M, g, g^{\prime}\right)$ be a compact connected complex manifold supplied with a pair of $h$-projectively equivalent Kähler metrics $g$ and $g^{\prime}$ that satisfy Assumption 1 . As shown in the previous section, $(M, g, \mathcal{F})$ is a Kähler-Liouville manifold whose partially ordered set consists of one element, and therefore $M$ is isomorphic to $\mathbb{C} P^{n}$ as a toric variety. In this case, $(M, g, \mathcal{F})$ is completely characterized by some invariants, several constants and a function on a circle (see [1, Section 7]). Here we assign to any triple $\left(M, g, g^{\prime}\right)$ a set of invariants, called fundamental invariants, which is an enlargement of the set of invariants stated above, and we show that those invariants characterize the triple $\left(M, g, g^{\prime}\right)$ uniquely up to isomorphisms. Let $\left(M, g, g^{\prime}\right)$ and $\left(N, s, s^{\prime}\right)$ be two such triples.

Definition 3.1. The triples $\left(M, g, g^{\prime}\right)$ and $\left(N, s, s^{\prime}\right)$ are called isomorphic if there is a biholomorphic diffeomorphism $\Phi: M \rightarrow N$ such that $\Phi^{*} s=g$ and $\Phi^{*} s^{\prime}=g^{\prime}$.

Let $\chi(c)=\operatorname{det}(\mathcal{A}-c \mathbf{1})$ (cf. (2.2) $)$ be associated to the triple $\left(M, g, g^{\prime}\right)$ and let $h_{1}, \ldots, h_{n}$ be as above. In view of (2.8), the functions $h_{i}$ here are the same as those appearing in [1, Proposition 1.12] and [1, Theorem 3.1], and where they were called fundamental functions 4 Therefore, by [1, Theorem 4.10] we have

Lemma 3.1. $\min h_{k}=\max h_{k+1}$ for $k=1, \ldots, n-1$.

\footnotetext{
${ }^{3} \mathrm{~A}$ local construction of these infinitesimal automorphisms in the more general case of Hermitian metrics is given in Section 4 of the present paper.

${ }^{4}$ Note however that the $h_{k}$ 's appearing in [1] Proposition 1.12] are defined uniquely up to an affine transform $h_{k} \mapsto \alpha h_{k}+\beta$, where $\alpha, \beta \in \mathbb{R}$ are independent of $k$.
} 
Define the positive numbers

$$
c_{0}:=\max _{M} h_{1}, \quad c_{k}:=\min _{M} h_{k}=\max _{M} h_{k+1}(1 \leq k \leq n-1), \quad c_{n}:=\min _{M} h_{n} .
$$

It is clear that $c_{0}>c_{1}>\cdots>c_{n}>0$.

Next, consider the level sets $L_{k} \stackrel{\text { def }}{=}\left\{\chi\left(c_{k}\right)=0\right\}(k=0, \ldots, n)$. By the results proved in [1] and summarized in [1, Section 7] we have

Lemma 3.2. (a) The $L_{k}$ are totally geodesic, complex hypersurfaces.

(b) By an identification of $M$ as a toric variety to the complex projective space $\mathbb{C} P^{n}$ with homogeneous coordinates $\left[z_{0}, \ldots, z_{n}\right]$, each $L_{k}$ identifies with the hypersurface $\left(\cong \mathbb{C} P^{n-1}\right)$ given by $z_{k}=0$.

(c) The $Y_{k}=J\left(\operatorname{grad} \chi\left(c_{k}\right)\right)(0 \leq k \leq n) \operatorname{span} \mathfrak{h}$.

(d) The $Y_{n}$ generate an effective circle action on $S=\bigcap_{k=1}^{n-1} L_{k} \simeq \mathbb{C} P^{1}$ with two fixed points $q_{1}=L_{0} \cap S$ and $q_{2}=L_{n} \cap S$.

Denote by $\gamma(t)\left(\|\dot{\gamma}(0)\|_{g}=1\right)$ a minimal length geodesic connecting $q_{1}$ with $q_{2}$. Let $l>0$ be the length of $\gamma$. (Notice that the points $t=0$ and $t=l$ are conjugate.) Consider the function

$$
h(t) \stackrel{\text { def }}{=} \chi\left(c_{n}\right)(\gamma(t))
$$

defined on the interval $[0, l]$.

Definition 3.2. The numbers $c_{0}>c_{1}>\cdots>c_{n}>0$, the length $l>0$, and the $C^{\infty}$ function $h$ on $[0, l]$ are called fundamental invariants of the triple $\left(M, g, g^{\prime}\right)$.

The main result of this section is

Theorem 3.3. The triples $\left(M, g, g^{\prime}\right)$ and $\left(N, s, s^{\prime}\right)$ are isomorphic if and only if their fundamental invariants coincide.

Proof. It follows from the definition of the fundamental invariants that isomorphic triples have the same fundamental invariants.

Let us prove the inverse statement. Suppose that $\left(M, g, g^{\prime}\right)$ and $\left(N, s, s^{\prime}\right)$ have the same fundamental invariants, namely $\left(\left\{c_{0}, \ldots, c_{n}\right\}, l, h(t)\right)$. Consider the KählerLiouville manifolds $(M, g, \mathcal{F})$ and $(N, s, \mathcal{H})$, where

$$
\mathcal{F}:=\operatorname{span}\left\{K_{c}\left(g, g^{\prime}\right) \mid c \in \mathbb{R}\right\} \quad \text { and } \quad \mathcal{H}:=\operatorname{span}\left\{K_{c}\left(s, s^{\prime}\right) \mid c \in \mathbb{R}\right\} .
$$

We have proved that $(M, g, \mathcal{F})$ and $(N, s, \mathcal{H})$ are Kähler-Liouville manifolds of type $(A)$ whose associated partially ordered sets consist of one element.

Lemma 3.4. The Kähler-Liouville manifolds $(M, g, \mathcal{F})$ and $(N, s, \mathcal{H})$ are mutually isomorphic.

Proof. By [1, Section 7, they are mutually isomorphic if and only if the corresponding elements $\left(\left\{\tilde{c}_{k}\right\}, \tilde{d}, \tilde{l}, \tilde{h}\right) \in \mathcal{C}_{n}$ are mutually equivalent (see [1, p. 134] for the set $\mathcal{C}_{n}$ and the equivalence). For $(M, g, \mathcal{F})$ one has $\tilde{l}=2 l, \tilde{c}_{k}=\left(c_{k}-c_{n}\right) /\left(c_{0}-c_{n}\right)$,

$$
\begin{aligned}
\tilde{h}(t) & =\left[\left(\tilde{h}_{1}(\gamma(t))-\tilde{c}_{n}\right) \ldots\left(\tilde{h}_{n}(\gamma(t))-\tilde{c}_{n}\right)\right] /\left[\left(\tilde{c}_{0}-\tilde{c}_{n}\right) \ldots\left(\tilde{c}_{n-1}-\tilde{c}_{n}\right)\right] \\
& =h(t) /\left[\left(c_{0}-c_{n}\right) \ldots\left(c_{n-1}-c_{n}\right)\right], \quad t \in[0, l],
\end{aligned}
$$

and $\tilde{h}(t)=\tilde{h}(-t), t \in[-l, 0]$, where $\tilde{h}_{k}:=\left(h_{k}-c_{n}\right) /\left(c_{0}-c_{n}\right)$. By Proposition $7.1(5)$ in [1], $\tilde{d}$ is determined uniquely from $\tilde{l}$ and $\tilde{h}$. Hence, $\left(\left\{\tilde{c}_{k}\right\}, \tilde{d}, \tilde{l}, \tilde{h}\right)$ are determined by the data $\left(\left\{c_{k}\right\}, l, h\right)$, and therefore coincide with those for $(N, s, \mathcal{H})$. 
By Lemma 3.4 there exists an isomorphism $\Phi:(M, g, \mathcal{F}) \rightarrow(N, s, \mathcal{H})$ of KählerLiouville manifolds. In particular, $\Phi^{*} s=g$. For the sake of notation, we denote the symbols for $\left(N, s, s^{\prime}\right)$ by attaching the hat ^ to the corresponding symbols for $\left(M, g, g^{\prime}\right)$, e.g., $\hat{h}_{k}, \hat{\tilde{h}}_{k}, \hat{L}_{k}$, etc. Using that the invariants $\left(\left\{\tilde{c}_{k}\right\}, \tilde{d}, \tilde{l}, \tilde{h}\right)$ and $\left(\left\{\hat{\tilde{c}}_{k}\right\}, \hat{\tilde{d}}, \hat{\tilde{l}}, \hat{\tilde{h}}\right)$ are not only mutually equivalent as elements of $\mathcal{C}_{n}$ but even coincide, i.e. $\tilde{h}(t)=\hat{\tilde{h}}(t), \tilde{l}=\hat{\tilde{l}}$, and $\tilde{d}=\hat{\tilde{d}}$, one sees from the construction of $\Phi$ in 11, Theorem 7.2$]$ that $\Phi^{*}\left(\hat{\tilde{h}}_{k}\right)=\tilde{h}_{k}$. As $\tilde{h}_{k}=\left(h_{k}-c_{n}\right) /\left(c_{0}-c_{n}\right)$ and $\hat{\tilde{h}}_{k}:=\left(\hat{h}_{k}-c_{n}\right) /\left(c_{0}-c_{n}\right)$ we obtain that

$$
\Phi^{*} \hat{h}_{k}=h_{k} \quad(1 \leq k \leq n) .
$$

Recall that $M^{1}$ is the subset of $M$ where the $h_{k}$ are all distinct and $d h_{k} \neq 0 . M^{1}$ is the same subset of $M$ as the one considered in [1, p. 85]. In particular, it is open and dense in $M$ by [1, Theorem 3.1] and, by (3.2), $\Phi\left(M^{1}\right)=N^{1}$. For each $p \in M^{1}$, let $D_{k}(p)$ be the eigenspace of the endomorphism $A$ on $T_{p} M$ corresponding to the eigenvalue $h_{k}(p)$. Note that the $D_{k}(p)$ 's are mutually orthogonal $J$-invariant subspaces of $T_{p} M$ of real dimension two. Using (2.7) one sees that $\left(\operatorname{grad} h_{k}\right)_{p} \in$ $D_{k}(p)$ and, by the $J$-invariance of $D_{k}(p),\left(J \operatorname{grad} h_{k}\right)_{p} \in D_{k}(p)$ as well. Recall that $\Phi$ preserves $J$ and $\Phi^{*} s=g$. This together with (3.2) and $\left(\operatorname{grad} h_{k}\right)_{p},\left(J \operatorname{grad} h_{k}\right)_{p} \in$ $D_{k}(p)$ shows that $\Phi$ maps $D_{k}(p)$ onto $\hat{D}_{k}(\Phi(p))$ for any $p \in M^{1}$. Hence, $\Phi$ maps the tensor field $A$ to the corresponding tensor field $\hat{A}$. Finally, in view of (2.1), $\Phi^{*} s^{\prime}=g^{\prime}$. This completes the proof of Theorem 3.3 .

\section{Local structure in the Hermitian Case}

Let $M$ be an $n$-dimensional complex manifold, and $g$ and $g^{\prime}$ be two Hermitian metrics on it. Suppose that the corresponding Levi-Civita connections $\nabla$ and $\nabla^{\prime}$ satisfy relation (1.2) in the Introduction; that is,

$$
\nabla_{X}^{\prime} Y-\nabla_{X} Y=\phi(X) Y+\phi(Y) X-\phi(P X) Q Y-\phi(P Y) Q X
$$

where $\phi$ is a one-form on $M, P$ is a $(1,1)$-type tensor field on $M$ which is nondegenerate and skew-symmetric with respect to both metrics $g$ and $g^{\prime}$, and $Q=$ $-P^{-1}$. Let $J$ be the complex structure of $M$, and let $A$ be as in the previous section, i.e., the $(1,1)$-type tensor field defined by the formula (2.1). As $P$, $Q$, and $J$, are skew-symmetric with respect to both $g$ and $g^{\prime}$ we see from (2.1) that

$$
P A=A P, \quad Q A=A Q, \quad J A=A J .
$$

As in the previous section, we make the following assumption.

Assumption 2. There is a point $p_{0} \in M$ such that $A$ has $n$ distinct eigenvalues at $p_{0}, h_{1}\left(p_{0}\right)>\cdots>h_{n}\left(p_{0}\right)>0$, and $d h_{i} \neq 0(1 \leq i \leq n)$ at $p_{0}$.

Let $U$ be a neighborhood of $p_{0}$ such that Assumption 2 holds for any $p \in U$. Choose an orthonormal frame $V_{1}, \ldots, V_{n}, J V_{1}, \ldots, J V_{n}$ on $U$ so that $D_{i}$, the subbundle of $T U$ spanned by $V_{i}$ and $J V_{i}$, is an eigenspace of $A$. Then we have

$$
T U=D_{1} \oplus \cdots \oplus D_{n} \quad \text { and }\left.\quad A\right|_{D_{i}}=h_{i} \mathbf{1}_{D_{i}}
$$

where $\mathbf{1}_{D_{i}}$ denotes the identity map on $D_{i}$. As $Q$ commutes with $A$,

$$
\left.Q\right|_{D_{i}}=\left.b_{i} J\right|_{D_{i}},
$$

where $b_{i}$ are some nonzero functions. In particular, $Q$ and $P$ commute with the complex structure $J$. 
Proposition 4.1. $\left.d h_{i}\right|_{D_{j}}=0$ if $i \neq j$.

Proof. By [10, Lemma 2, II B],

$$
\begin{aligned}
-g\left(\left(\nabla_{X} A\right) Y, Z\right)= & \phi(A Y) g(X, Z)+\phi(A Z) g(X, Y) \\
& -\phi(A P Y) g(Q X, Z)-\phi(A P Z) g(Q X, Y) .
\end{aligned}
$$

Using (4.2) one obtains from (4.3) that $g\left(\left(\nabla_{V_{j}} A\right) V_{i}, V_{i}\right)=0$ for $i \neq j$. On the other hand, by differentiating the equality $A V_{i}=h_{i} V_{i}$ covariantly in the direction of $V_{j}$, and by using that

$$
2 g\left(\nabla_{V_{j}} V_{i}, V_{i}\right)=g\left(\nabla_{V_{j}} V_{i}, V_{i}\right)+g\left(V_{i}, \nabla_{V_{j}} V_{i}\right)=\nabla_{V_{j}}\left[g\left(V_{i}, V_{i}\right)\right]=0,
$$

one gets that $g\left(\left(\nabla_{V_{j}} A\right) V_{i}, V_{i}\right)=V_{j} h_{i}$. Hence, $d h_{i}\left(V_{j}\right)=0$. In a similar way one gets $d h_{i}\left(J V_{j}\right)=0$.

Since $d h_{i} \neq 0$ is assumed, we have $\left.d h_{i}\right|_{D_{i}} \neq 0$. Therefore there is a particular choice of $V_{i}$ so that

$$
d h_{i}\left(J V_{i}\right)=0 \quad(1 \leq i \leq n) .
$$

From now on we assume that the $V_{i}$ 's are chosen in this way. Put

$$
D^{+}:=\operatorname{span}\left\{V_{1}, \ldots, V_{n}\right\} \text { and } D^{-}:=\operatorname{span}\left\{J V_{1}, \ldots, J V_{n}\right\} \text {. }
$$

Note that $d_{p} h_{1}, \ldots, d_{p} h_{n}$ are linearly independent for any $p \in U$, and by Proposition 4.1 and (4.5), $D^{-}$is completely integrable.

Lemma 4.2. For $i \neq j$,

$$
\begin{aligned}
& \nabla_{V_{j}} V_{i}=\frac{1}{2\left(h_{i}-h_{j}\right)}\left(V_{i} h_{i}\right) V_{j}, \quad \nabla_{V_{j}}\left(J V_{i}\right)=\frac{1}{2\left(h_{i}-h_{j}\right)} \frac{b_{j}}{b_{i}}\left(V_{i} h_{i}\right) J V_{j}, \\
& \nabla_{J V_{j}} V_{i}=\frac{1}{2\left(h_{i}-h_{j}\right)}\left(\left(V_{i} h_{i}\right) J V_{j}-\frac{b_{i}}{b_{j}}\left(V_{j} h_{j}\right) J V_{i}\right), \\
& \nabla_{J V_{j}}\left(J V_{i}\right)=\frac{1}{2\left(h_{i}-h_{j}\right)}\left(-\frac{b_{j}}{b_{i}}\left(V_{i} h_{i}\right) V_{j}+\frac{b_{i}}{b_{j}}\left(V_{j} h_{j}\right) V_{i}\right) .
\end{aligned}
$$

Proof. Using (4.5) one obtains from (4.3) that

$$
\left(\nabla_{V_{j}} A\right) V_{i}=-\phi\left(A V_{i}\right) V_{j}+\phi\left(A P V_{i}\right) b_{j} J V_{j} .
$$

By formula (6) in [10, II B], $\phi(X)=-\frac{1}{4} d(\log \operatorname{det} A)(X)$. This together with (4.8) shows that $\left(\nabla_{V_{j}} A\right) V_{i}=\frac{1}{2}\left(V_{i} h_{i}\right) V_{j}$. On the other side, a covariant differentiation of the equality $\left(A-h_{i}\right) V_{i} \equiv 0$ in the direction of $V_{i}$ leads to $\left(\nabla_{V_{j}} A\right) V_{i}=$ $-\left(A-h_{i}\right) \nabla_{V_{j}} V_{i}$. Hence, $-\left(A-h_{i}\right) \nabla_{V_{j}} V_{i}=\frac{1}{2}\left(V_{i} h_{i}\right) V_{j}$, and therefore

$$
\nabla_{V_{j}} V_{i} \equiv \frac{1}{2\left(h_{i}-h_{j}\right)}\left(V_{i} h_{i}\right) V_{j} \quad \bmod D_{i}
$$

In a similar way one obtains the other formulas of the lemma modulo $D_{i}$. To prove the exact equalities, we note that $\nabla_{J V_{j}}\left(J V_{i}\right)$ is orthogonal to $J V_{i}$ (cf. (4.4)). Using this and the fact that (4.7) holds modulo $D_{i}$ we see that $\nabla_{J V_{j}}\left(J V_{i}\right)$ is a linear combination of $V_{j}$ and $V_{i}$, and so is $\left[J V_{j}, J V_{i}\right]=\nabla_{J V_{j}}\left(J V_{i}\right)-\nabla_{J V_{i}}\left(J V_{j}\right)$. As $D^{-}$is completely integrable, the Lie bracket $\left[J V_{j}, J V_{i}\right]$ is a section of $D^{-}$. Thus we have $\left[J V_{j}, J V_{i}\right]=0, \nabla_{J V_{j}}\left(J V_{i}\right)=\nabla_{J V_{i}}\left(J V_{j}\right)$, and

$$
\nabla_{J V_{j}}\left(J V_{i}\right)=\frac{1}{2\left(h_{i}-h_{j}\right)}\left(-\frac{b_{j}}{b_{i}}\left(V_{i} h_{i}\right) V_{j}+\frac{b_{i}}{b_{j}}\left(V_{j} h_{j}\right) V_{i}\right) .
$$


As $\nabla_{J V_{j}} V_{i}$ is orthogonal to $V_{i}$ and as $g\left(\nabla_{J V_{j}} V_{i}, J V_{i}\right)=-g\left(V_{i}, \nabla_{J V_{j}} J V_{i}\right)$ we see that (4.6) holds. In particular, $\nabla_{J V_{i}} V_{j}$ is a linear combination of $J V_{j}$ and $J V_{i}$. Differentiating $h_{i}$ in the direction of

$$
\left[J V_{i}, V_{j}\right]=\nabla_{J V_{i}} V_{j}-\nabla_{V_{j}}\left(J V_{i}\right),
$$

we have $0=\left(J V_{i}\right) V_{j} h_{i}-V_{j}\left(J V_{i}\right) h_{i}=-\left(\nabla_{V_{j}}\left(J V_{i}\right)\right) h_{i}$. This implies that the $D_{i^{-}}$ component of the derivative $\nabla_{V_{j}}\left(J V_{i}\right)$ vanishes. In particular, one has $g\left(\nabla_{V_{j}} V_{i}, J V_{i}\right)$ $=-g\left(V_{i}, \nabla_{V_{j}} J V_{i}\right)=0$. Hence, the $D_{i}$-component of $\nabla_{V_{j}} V_{i}$ vanishes as well.

Put

$$
W_{i}:=\sqrt{(-1)^{n-i} \prod_{k \neq i}\left(h_{k}-h_{i}\right)} V_{i} \quad(1 \leq i \leq n) .
$$

Proposition 4.3. (1) For $i \neq j$,

$$
\left[W_{i}, W_{j}\right]=\left[J W_{i}, J W_{j}\right]=\left[W_{i}, J W_{j}\right]=0,
$$

$$
\begin{aligned}
{\left[W_{i}, J W_{i}\right] } & =\sum_{k \neq i} \frac{\left|\prod_{l \neq i}\left(h_{l}-h_{i}\right)\right|}{\left|\prod_{l \neq k}\left(h_{l}-h_{k}\right)\right|} \frac{1}{h_{i}-h_{k}} \frac{b_{i}}{b_{k}}\left(W_{k} h_{k}\right) J W_{k} \\
& -\left(\sum_{l \neq i} \frac{W_{i} h_{i}}{h_{l}-h_{i}}+W_{i} \log \left|\frac{b_{j}}{b_{i}}\right|+\frac{W_{i}^{2} h_{i}}{W_{i} h_{i}}\right) J W_{i},
\end{aligned}
$$

where $j(\neq i)$ is arbitrary.

Proof. Item (1) is an immediate consequence of Lemma 4.2 and $\nabla_{X} Y-\nabla_{Y} X=$ $[X, Y]$. Using Lemma 4.2 and $g\left(\left[V_{i}, J V_{i}\right], Z\right)=g\left(\nabla_{V_{i}} J V_{i}, Z\right)-g\left(\nabla_{J V_{i}} V_{i}, Z\right)=$ $g\left(V_{i}, \nabla_{J V_{i}} Z\right)-g\left(J V_{i}, \nabla_{V_{i}} Z\right)$ for any $Z$ from the orthonormal frame, we get

$$
\left[V_{i}, J V_{i}\right] \equiv \sum_{k \neq i} \frac{1}{h_{i}-h_{k}} \frac{b_{i}}{b_{k}}\left(V_{k} h_{k}\right) J V_{k} \quad \bmod D_{i} .
$$

Hence, by (4.11), $\left[W_{j}, J W_{j}\right]=c_{j} W_{j}+\sum_{k=1}^{n} e_{j k} J W_{k}$, where $e_{j k} \neq 0$ for $j \neq k$. Using item (1) and the Jacobi identity for the Lie bracket of vector fields one obtains for $i \neq j$,

$$
\begin{aligned}
0 & =\left[W_{i},\left[W_{j}, J W_{j}\right]\right]=\left(W_{i} c_{j}\right) W_{j}+\sum_{k}\left(W_{i} e_{j k}\right) J W_{k}+e_{j i}\left[W_{i}, J W_{i}\right] \\
& =\left(W_{i} c_{j}\right) W_{j}+e_{j i} c_{i} W_{i}+\sum_{k}\left(W_{i} e_{j k}+e_{j i} e_{i k}\right) J W_{k} .
\end{aligned}
$$

By comparing both sides of the last equality we see that $c_{l}=0$ and $W_{i} e_{j k}=-e_{j i} e_{i k}$ for $i \neq j$. In particular, $e_{i i}=-W_{i} \log \left|e_{j i}\right|, j \neq i$. This together with (4.11) and (4.12) implies (2).

Corollary 4.4. The subbundle $D^{+}$is completely integrable, and its integral manifolds are totally geodesic.

Proof. Since by Lemma 4.2, $\nabla_{V_{j}} V_{i} \in D^{+}(j \neq i)$, it is enough to show that $\nabla_{V_{i}} V_{i} \in$ $D^{+}$. One has $g\left(\nabla_{V_{i}} V_{i}, J V_{j}\right)=-g\left(V_{i}, \nabla_{V_{i}}\left(J V_{j}\right)\right)$. If $i \neq j$, the right-hand side vanishes by Lemma 4.2, If $i=j, g\left(V_{i}, \nabla_{V_{i}}\left(J V_{i}\right)\right)=g\left(V_{i},\left[V_{i}, J V_{i}\right]\right)$, which vanishes by Proposition 4.3 (2). 
Let $W_{1}^{*}, \ldots, W_{n}^{*},\left(J W_{1}\right)^{*}, \ldots,\left(J W_{n}\right)^{*}$ be the dual frame to $W_{1}, \ldots, W_{n}, J W_{1}, \ldots, J W_{n}$. Then it follows from Proposition 4.3 (1) that the one-forms $W_{i}^{*}$ are closed. Therefore there is a system of functions $\left(w_{1}, \ldots, w_{n}\right)$ such that $W_{i}^{*}=d w_{i}$ on a neighborhood of $p_{0}$. The function $h_{i}$ is thus a function of $w_{i}$ alone. More generally, a function $u$ satisfying $\left.d u\right|_{D^{-}}=0$ is a function of $\left(w_{1}, \ldots, w_{n}\right)$. For simplicity we will write $\partial_{i} u$ instead of $W_{i} u$.

Proposition 4.5. (1) $\left.d\left(b_{i} / b_{j}\right)\right|_{D^{-}}=0$ for any $i, j$.

(2) There are nonzero functions $\hat{b}_{i}\left(w_{i}\right)(1 \leq i \leq n)$ such that

$$
b_{1} / \hat{b}_{1}=\cdots=b_{n} / \hat{b}_{n} .
$$

Proof. By Proposition $4.3(2),\left[W_{i}, J W_{i}\right]=\sum_{k} e_{i k} J W_{k}$. Proposition 4.3 (1) and Jacobi's identity imply that

$$
\left(J W_{j}\right) e_{i k}=0 \quad(j \neq i) .
$$

This together with the explicit formulas for the $e_{i k}$ 's from Proposition 4.3 (2) show that $\left(J W_{j}\right)\left(b_{i} / b_{k}\right)=0$ for $j \neq i$. Taking $k=j$ in the last formula and then replacing $i$ by $k$ and $j$ by $i$, one sees that $\left(J W_{i}\right)\left(b_{i} / b_{k}\right)=0$ as well. Therefore,

$$
\left.d\left(b_{i} / b_{k}\right)\right|_{D^{-}}=0 .
$$

To prove $(2)$, recall that $\partial_{j} e_{i k}=-e_{i j} e_{j k}(j \neq i)$, and hence

$$
-\partial_{j} \log \left|e_{i k}\right|=e_{i j} e_{j k} / e_{i k}, \quad j \neq i, i \neq k .
$$

Using (4.13) and the explicit formulas for the $e_{i k}$ 's we see that, if $n \geq 3$ and $j \neq i, k$,

$$
\partial_{j} \log \left|b_{i} / b_{k}\right|=0 \quad \text { and } \quad \partial_{j} \log \left|b_{i} / b_{j}\right|=\partial_{j} \log \left|b_{k} / b_{j}\right| .
$$

The former formula shows that $b_{i} / b_{k}$ depends only on $\left(w_{i}, w_{k}\right)$. Hence by the latter formula,

$$
\partial_{i} \partial_{j} \log \left|b_{i} / b_{j}\right|=0
$$

If $n=2$, by comparing both sides of $\partial_{j} e_{i i}=-e_{i j} e_{j i}$, we conclude that (4.14) still holds. Hence, there are nonzero functions $\hat{b}_{j}\left(w_{j}\right)(1 \leq j \leq n)$ such that

$$
\partial_{j} \log \left|b_{i} / b_{j}\right|=-\partial_{j} \log \left|\hat{b}_{j}\right|
$$

for any $i \neq j$. Then

$$
\mu_{i j}=\log \left|b_{i} / b_{j}\right|-\log \left|\hat{b}_{i} / \hat{b}_{j}\right|
$$

are constants that satisfy $\mu_{j i}=-\mu_{i j}$ and $\mu_{i j}+\mu_{j k}=\mu_{i k}$. Putting $\nu_{i}=(1 / n) \sum_{k} \mu_{i k}$ we have $\mu_{i j}=\nu_{i}-\nu_{j}$. Hence, redefining $\hat{b}_{i}$ as $\exp \left(\nu_{i}\right) \hat{b}_{i}$, we get from (4.15) that $\mu_{i j}=0$. Finally, choosing appropriately the signs of the $\hat{b}_{i}$ 's we obtain (2).

Consider the vector fields $Y_{1}, \ldots, Y_{n}$ defined by

$$
\sum_{k=1}^{n}\left(-h_{i}\right)^{k-1} Y_{k}=(-1)^{n-i} \frac{W_{i} h_{i}}{\hat{b}_{i}} J W_{i}
$$

Theorem 4.6. (1) Each $Y_{k}$ is an infinitesimal automorphism of the Hermitian manifold $M$, i.e., $\mathcal{L}_{Y_{k}} g=0, \mathcal{L}_{Y_{k}} J=0$.

(2) $\left[Y_{i}, Y_{j}\right]=0$ for any $i, j$.

(3) $\left\{K_{i}, Y_{j}\right\}=0$ for any $i, j$. 
Proof. We write $\sum_{k} c_{i k} Y_{k}=J W_{i}$. Since by (4.16), $d c_{i k}=0$ on $D^{-}$, it follows that

$$
0=\left[J W_{i}, J W_{j}\right]=\sum_{k, l} c_{i k} c_{j l}\left[Y_{k}, Y_{l}\right] .
$$

Hence (2) holds. Using (2), $Y_{j} c_{i k}=0$, and $J W_{k}=\sum_{j} c_{k j} Y_{j}$, we see that $\left[Y_{i}, J W_{k}\right]=$ 0 for any $i, k$. Next, we show that $\left[Y_{i}, W_{k}\right]=0$ for any $i, k$. Since $W_{j} c_{i k}=0$ for $j \neq i$, we obtain as above

$$
\sum_{k} c_{i k}\left[W_{j}, Y_{k}\right]=\left[W_{j}, J W_{i}\right]=0 \quad(j \neq i) .
$$

We have also

$$
\sum_{k}\left(W_{i} c_{i k}\right) Y_{k}+\sum_{k} c_{i k}\left[W_{i}, Y_{k}\right]=\left[W_{i}, J W_{i}\right]=\sum_{j} e_{i j} J W_{j}=\sum_{j, k} e_{i j} c_{j k} Y_{k},
$$

where $e_{i j}$ are as in the proof of Proposition 4.5. On the other side, a direct computation involving Proposition 4.5(2) and the explicit expressions for the $e_{i j}$ 's and the $c_{i k}$ 's shows that

$$
W_{i} c_{i k}=\sum_{j} e_{i j} c_{j k}
$$

Therefore, from (4.18), $\sum_{k} c_{i k}\left[W_{i}, Y_{k}\right]=0$. This combined with (4.17) implies that $\left[W_{i}, Y_{k}\right]=0$.

Hence, we have shown that $\left[Y_{k}, W_{i}\right]=\left[Y_{k}, J W_{i}\right]=0$ for any $i, k$. This implies that $\mathcal{L}_{Y_{k}} J=0$ and by formula (2.6),$\sum_{j=1}^{n}\left(-h_{i}\right)^{j-1}\left\{Y_{k}, K_{j}\right\}=0$ for any $i, k$. Thus $\mathcal{L}_{Y_{k}} K_{j} \equiv\left\{Y_{k}, K_{j}\right\}=05$ Since $(1 / 2) K_{n}$ is the Hamiltonian of the geodesic flow, we also have $\mathcal{L}_{Y_{k}} g=0$.

Next we show that the local structure of $M$ is completely characterized by Theorem 4.6 ,

Trivialization of the local structure: Let $U$ be an open set of $\mathbb{C}^{n}$ with coordinates $\left\{\left(z_{1}, \ldots, z_{n}\right)\right\}$. Writing $z_{i}=x_{i}+\sqrt{-1} y_{i}$, we put

$$
X_{i}=\frac{\partial}{\partial x_{i}}, \quad Y_{i}=\frac{\partial}{\partial y_{i}} .
$$

Then $J X_{i}=Y_{i}$, where $J$ denotes the standard complex structure of $\mathbb{C}^{n}$. Suppose that $U$ is of the form

$$
U=\left\{\left(z_{1}, \ldots, z_{n}\right) \mid\left(x_{1}, \ldots, x_{n}\right) \in U_{0},\left(y_{1}, \ldots, y_{n}\right) \in V_{0}\right\} \simeq U_{0} \times V_{0},
$$

where $U_{0}$ and $V_{0}$ are open neighborhoods of 0 in $\mathbb{R}^{n}$. Let $U_{1}$ be an open disk in $\mathbb{R}^{n}=\left\{\left(w_{1}, \ldots, w_{n}\right)\right\}$, and let $h_{i}\left(w_{i}\right)$ and $\hat{b}_{i}\left(w_{i}\right)(1 \leq i \leq n)$ be functions of a single variable such that

$$
h_{1}>\cdots>h_{n}>0, \quad d h_{i} \neq 0, \quad \hat{b}_{i} \neq 0
$$

on $U_{1}$. Define the vector fields $\tilde{X}_{i}(1 \leq i \leq n)$ by

$$
\sum_{k=1}^{n}\left(-h_{i}\right)^{k-1} \tilde{X}_{k}=(-1)^{n-i} \frac{1}{\hat{b}_{i}} \frac{\partial h_{i}}{\partial w_{i}} \frac{\partial}{\partial w_{i}} .
$$

Note that $\left[\tilde{X}_{i}, \tilde{X}_{j}\right]=0$ for any $i, j$. Therefore, taking a reference point $p_{0} \in U_{1}$, we get a unique diffeomorphism $\Phi: U_{1} \rightarrow U_{0}$ so that $\Phi\left(p_{0}\right)=0$ and $\Phi_{*}\left(\tilde{X}_{i}\right)=X_{i}$

${ }^{5}$ We identify the symmetric $(m, 0)$-type tensors with the corresponding functions on $T^{*} M$. 
for any $i$. Put $\Phi_{*}\left(\partial / \partial w_{i}\right)=W_{i}$, and regard $h_{i}$ and $\hat{b}_{i}$ as functions on $U_{0}$ via the diffeomorphism $\Phi$. We define the functions $K_{i}$ on the cotangent bundle $T^{*} U$ by the formula

$$
\sum_{j=1}^{n}\left(-h_{i}\right)^{j-1} K_{j}=(-1)^{n-i}\left(W_{i}^{2}+\left(J W_{i}\right)^{2}\right) .
$$

It follows from our construction that the vector fields $Y_{k}$ satisfy (4.16) and commute with $W_{i}$ and $J W_{i}$. Hence $Y_{k}$ commutes with $K_{j}$. It is easily seen that the Lie brackets $\left[W_{i}, J W_{j}\right]$ vanish for any $i \neq j$ (cf. (4.17)). Hence the $K_{i}$ are mutually Poisson commuting. Let $g$ be the Hermitian metric on $U$ so that $(1 / 2) K_{n}$ is the Hamiltonian of its geodesic flow. Then it follows that the $Y_{k}$ are infinitesimal holomorphic isometries of the Hermitian manifold $(U, g)$. Define the $(1,1)$-type tensor fields $A$ and $Q$ by

$$
A J=J A, \quad Q J=J Q, \quad A W_{i}=h_{i} W_{i}, \quad Q W_{i}=\hat{b}_{i} J W_{i},
$$

and put $P=-Q^{-1}$. Define the Hermitian metric $g^{\prime}$ by the relation (2.1). Then one verifies that the corresponding covariant derivatives $\nabla$ and $\nabla^{\prime}$ satisfy (1.2).

The range of the hierarchy: We refer to [10, IID] for the definition and the main properties of the hierarchy of pairs of metrics corresponding to a given pair of $P Q^{\epsilon}$-projectively equivalent metrics $g$ and $g^{\prime}$. Starting with a pair of $h$-projectively equivalent Kähler metric $\$ 6$ one gets a hierarchy of pairs of Hermitian metrics satisfying equation (1.2) with $P=J \cdot \alpha(A)$ and $Q=\alpha(A)^{-1} \cdot J$, where $\alpha:(a, b) \rightarrow \mathbb{R}$, $(a, b) \subseteq \mathbb{R}$, is a real-analytic function such that $\alpha(A)$ is well-defined and nondegenerate on $M$.

Following the trivialization construction discussed above we choose $h_{i}\left(w_{i}\right)$ and $\hat{b}_{i}\left(w_{i}\right)(1 \leq i \leq n)$ so that $h_{1}>\cdots>h_{n}>0, d h_{i} \neq 0$, and $\hat{b}_{i} \neq 0$. Assume for simplicity that $U_{1}=\left\{\left(w_{1}, \ldots, w_{n}\right) \mid w_{i} \in(-\delta, \delta), 1 \leq i \leq n\right\}, \delta>0$, and that the $h_{i}$ 's have positive first derivatives on $(-\delta, \delta)$. Consider the functions $\alpha_{j}:(-\delta, \delta) \rightarrow \mathbb{R}$,

$$
\alpha_{i}:=\hat{b}_{i} \circ h_{i}^{-1}, \quad 1 \leq i \leq n,
$$

and assume that there exist $1 \leq k, l \leq n$ so that $\alpha_{k} \not \equiv \alpha_{l}$. Note that the latter assumption implies that the pairs of metrics obtained from $h_{i}$ and $\hat{b}_{i}$ via the trivialization construction do not belong to the hierarchy obtained from a pair of $h$-projectively equivalent Kähler metrics. One sees from (4.20) that these pairs of metrics can be parametrized by $2 n$ functional parameters.

Remark. As a matter of fact, arguing similarly, one can construct global examples of such pairs of metrics on $\mathbb{C} P^{n}$ by modifying the examples considered in [2], [10, VI]. In particular, there are many examples of pairs of Hermitian metrics $\left(g, g^{\prime}\right)$ on $\mathbb{C} P^{n}$ satisfying (1.2) beyond the hierarchies.

\section{REFERENCES}

[1] K. Kiyohara, Two classes of Riemannian manifolds whose geodesic flows are integrable, Mem. Amer. Math. Soc., 130 (1997), no. 619, viii+143 pp. MR1396959 (98f:58150)

[2] M. Igarashi and K. Kiyohara: On Hermite-Liouville manifolds, to appear in J. Math. Soc. Japan.

[3] K. Kiyohara: On Kähler-Liouville manifolds, Differential geometry and integrable systems (Tokyo, 2000), 211-222, Contemp. Math., 308, AMS, Providence, RI, 2002. MR1955637 (2003k:53113)

${ }^{6}$ Then $P=Q=J$ and $\epsilon=-1$. 
[4] T. Ōtsuki and Y. Tashiro: On curves in Kählerian spaces, Math. J. Okayama Univ., 4 (1954), 57-78. MR0066024(16:521d)

[5] V. Matveev and P. Topalov: Trajectory equivalence and corresponding integrals, Regular and Chaotic Dynamics, 3 (1998), no. 2, 30-45. MR.1693470 (2000d:37068)

[6] V. Matveev and P. Topalov: Quantum integrability of Beltrami-Laplace operator as geodesic equivalence, Math. Z., 238 (2001), no. 4, 833-866. MR1872577 (2002k:58068)

[7] Y. Tashiro: On a holomorphically projective correspondence in an almost complex space, Math. J. Okayama Univ., 6 (1957), 147-152. MR0087181 (19:316b)

[8] P. Topalov: Hierarchy of integrable geodesic flows, Publicacions Matemàtiques, 44 (2000), no. 1, 257-276. MR1775764 (2001g:37104)

[9] P. Topalov: Geodesic hierarchies and involutivity, J. Math. Phys., 42 (2001), no. 8, 38983914. MR1845225 (2002e:37096)

[10] P. Topalov: Geodesic compatibility and integrability of geodesic flows, J. Math. Phys., 44 (2003), no. 2, 913-929. MR.1953103 (2003j:37088)

[11] K. Yano and H. Hiramatu: Kaehlerian manifolds with constant scalar curvature admitting a holomorphically projective vector field, J. Differential Geometry, 14 (1979), 81-92. MR577880 (81f:53054)

[12] K. Yano and H. Hiramatu: Isometry of Kaehlerian manifolds to complex projective spaces, J. Math. Soc. Japan, 33 (1981), no. 1, 67-78. MR.597481 (82g:53075)

Department of Mathematics, Okayama University, 3-1-1 Tsushima-naka, Okayama, 700-8530 JAPAN

Department of Mathematics, Northeastern University, 360 Huntington Avenue, Boston, Massachusetts 02115

E-mail address: p.topalov@neu.edu 